\title{
Smart Packaging in Intralogistics: An Evaluation Study of Human- Technology Interaction in Applying New Collaboration Technologies
}

\author{
Benedikt Mättig \\ Fraunhofer Institute for Material Flow and Logistics \\ IML, Dortmund, Germany \\ Benedikt.Maettig@iml.fraunhofer.de
}

\author{
Veronika Kretschmer \\ Fraunhofer Institute for Material Flow and Logistics \\ IML, Dortmund, Germany \\ Veronika.Kretschmer@iml.fraunhofer.de
}

\begin{abstract}
Handling and packaging of heterogeneous products with different weights and sizes with optimal packaging schemes is a challenging task for the e-commerce industry. Furthermore, to keep the packaging process on a standardized level independent of the experience level of the employee, the demand of digital humancentered solutions is increasing. Against this background, two different digital assistance systems to indicate packaging order and scheme - Augmented Reality (AR) based data glasses and a LED based packaging assistant - were developed. In a laboratory study the interaction between human and both digital devices regarding subjective workload, usability, user experience, physical complaints and objective measurements was evaluated - with a conventional paper list as control group. Results indicate that both the AR and LED interface are appropriate solutions to assist warehouse workers in packaging. However, it can be supposed that the LED interface seems to be a better method in terms of physical and especially visual strains.
\end{abstract}

\section{Introduction}

\subsection{Motivation}

In general, the role of intralogistics is growing in importance. Along with rising globalization and flexibilization of labor markets and the ensuring modern requirements of logistics, logistics systems need to become more flexible, efficient and customerfocused [1]. Logistics warehouses differ with regard to their size and available machinery according to the respective sector, the products to be handled and the size of the company. In recent years, the e-commerce industry achieved large increases in sales and turn-over [2]. Therefore, this sector experiences a large growth rate which on the other hand also put pressure on these businesses. After all, a main priority for e-commerce is to send out orders to their customers as quickly as possible. A special characteristic about orders in ecommerce business is that an order usually contains one to several heterogeneous products with different weights and sizes. This leads to a great challenge for order picking and the process of packaging. Poorly packaged orders usually take up more space in the truck and result in both higher costs and a worse ecobalance. Therefore, assistants are needed to support the optimization of packaging.

In the working world several new technological assistance systems become more and more common to reorganize and optimize working processes and support employees. Especially in the field of intralogistics, new information and collaboration technologies were introduced along with increasing digitization and automation of processes within the increasingly networked economy. As manual work activities like transporting, sorting, storing, picking, packing and distributing cannot be fully replaced by technologies, the role of the human being will remain important in future.

It can be assumed that the cooperation between technical assistance systems and human beings within a so-called »Social Networked Industry « will evoke a change in psychological and especially cognitive demands during communication and interaction with the autonomously interacting cyberphysical systems of an industry 4.0 [3]. A »Social Networked Industry« stands for industrially oriented forms of social networks, in which people and cyberphysical systems in the companies cooperate with each other (vertical networking), but also for new forms of networking, in which companies cooperate extensively with one another (horizontal networking) [3]. All participants within this collaboration will be organized in a social network to enable machines speak to humans with natural language. Thus, collaboration technologies will become one key enabler for the cooperation between humans and machines. The implementation of humanmachine collaboration in intralogistic locations 
provides the basis for using a technical assistance system that can react to both given and varying restrictions by the employee or environmental conditions.

Employees in German warehouses were subject to various physical and mental demands or job-related environmental factors [4]. Nowadays, it is presumed that new forms of load patterns will occur as a result of digitization. We conclude that one challenging task of companies is to adapt technologies to employees' needs and integrate employees into complex work environments that are changing rapidly and continuously.

In order to understand interaction between new technologies and human workers in intralogistics the new research topic »Cognitive Ergonomics« was developed. It evolves as an important field in evaluating human-machine interfaces with respect to regarding various human-centered factors such as mental workload, usability or user experience [4]. The main objective is to create a load-optimized interaction between technical assistance systems and their operators to assist warehouse workers by new technologies while handling economic goods. To achieve this, both laboratory and field studies have been conducted to gain new insights. This paper presents an evaluation study on human factors while interacting with new collaboration technologies for intralogistics. Furthermore, it gives recommendations for the purposeful use and cognitive-ergonomic design of smart devices in intralogistics.

\subsection{State of the Art}

The digitization of logistics is still far behind initial expectations. Most processes are handled manually or in a paper-based way [5]. This factor also has significant influence on the distribution and use of digital assistants in the supply chain. Currently, digital assistants are mostly used in the area of order picking. There are various solutions that support employees in the picking process like pick-by-light, pick-by-vision or pick-by-voice [6]. Other processes in logistics are usually characterized by very simple activities. Employees make up for the lack of support through their personal experience. Also the packaging process is mostly executed based on personal experience while it is based on the bin packing problem which is NPhard (so-called non-deterministic polynomial-time hardness) [7]. To compensate for fluctuations in packaging and to increase packaging quality, a software solution is therefore required for optimization.

There are some software systems that can for example calculate optimal packaging schemes. There are e.g. software solutions which are using different parameters and machine learning attempts to solve heterogeneous packaging schemes [7]. These software solutions consider problems like load balancing and constraints of stability. Research has also been carried out to guide the employee through a previously calculated scheme.

The AR technology has recently been the focus of prominent research in educational research. An overview article makes clear that the AR application has been used in educational contexts such as humanities and arts, eHealth, engineering, manufacturing and construction and science so far [8]. Since AR can demonstrably create interactive learning environments, the application is targeted in the logistics context to support intralogistic work processes. In the course of this, several studies in logistics research have already been carried out to investigate the general suitability of AR for packaging $[9,10,11]$. Study results show that an AR device is well suited to support operational logistics staff in palletizing, even if usability could still be improved [11]. Furthermore, research results clarify that AR offers opportunities to plan logistics systems more flexibly and supports planners in reacting more efficiently to rapidly changing market requirements [1]. Apart from this, there are currently no industrysuitable and intuitive solutions on the market for guiding employees through the packaging process. Industrial AR applications are currently used in the following areas: product design, plant design, training of production processes, production assistance, quality assurance, production logistics or remote maintenance [12].

In current logistics environments the packaging task is executed according to the knowledge, assessment and experience of the employee. But especially in logistics, new workers often enter a company that may not have any packaging expertise. Therefore, companies might not have enough human resources to train new staff. Based on these circumstances and in order to bridge the knowledge gap between different workers there is the need for efficient and intuitive packaging assistants for logistics systems.

\section{Digital assistants for manual packaging}

As part of a federal research project which addresses human factors in the digitized industrial world and new solutions for human-machine interaction, two different digital assistance systems for the process of manual packaging were developed by the authors of this paper. Both solutions try to realize an intuitive and easy-to-use solution for the 
visualization of calculated packaging schemes. Both pursue different ways to enable the targeted solution. The main target of both solutions was to minimize the mental workload of the worker and to optimize the packaging result regarding volume, time and error rate. The focus of the development was on creating an intuitive and easy-to-understand way of information visualization. Both solutions use a minimal amount of information to keep the assistant simple and reduce misunderstandings. Both assistants are based on the same process of placing heterogeneous articles inside a predefined delivery box. Beforehand, an optimizing software calculated an optimal packaging scheme for the articles and the size of the delivery box to reduce the empty space inside the box and to maximize the degree of filling.

In the development of both assistants, different approaches of provisioning information are used. While one solution is technically much more complex and embeds virtual information directly into reality, the other solution is based on inexpensive and simple elements for presenting information.

\subsection{Augmented Reality assistant}

The first packaging assistant makes use of a visual connection between objects and their corresponding information. This approach is based on the law of proximity, i.e. objects that are visually adjacent are interpreted as belonging together [14].

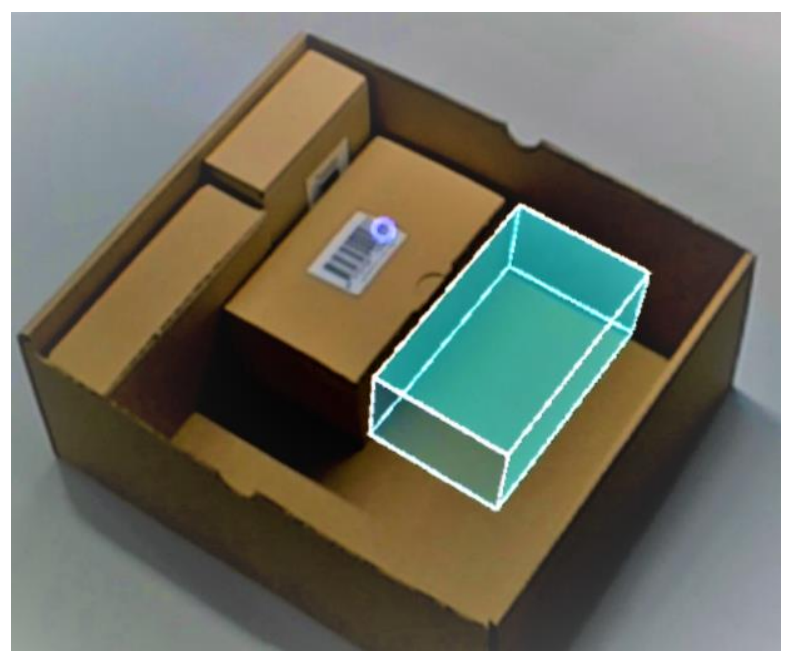

Figure 1. View through the Hololens

As a result, information is assigned directly to the corresponding physical object when both are visually connected. In this case, this is achieved through the use of data glasses and AR. The developed assistant uses the Microsoft Hololens which is able to visualize virtual elements directly embedded inside the real world. Due to the powerful sensor technology of the glasses, holograms appear fixed in the room and enable a realistic representation of virtual objects. Through a software which was designed as part of the assistant, the packaging employee receives step-by-step instructions and is guided through the process. In the course of this, the glasses indicate which product has to be packaged and provide feedback as to whether the correct product was selected. By means of a scanning glove and a small clicker the worker is able to interact with the glasses. With the glove he scans the article which has to be placed in the delivery box. After the article was scanned, the glasses show a virtual hologram embedded into the reality where the article has to be placed in the box (see Figure 1). After placing the article, the worker confirms the placement by using the clicker.

For reasons of usability, no gestures or voice input was used. In the run-up of the development, various input method tests were carried out. These showed that both gestures and voice input are too unreliable for the considered scenario. Another advantage of using the clicker and the glove for the interaction was that both systems give a haptic feedback to the user. Latest research suggests that the effects of haptic feedback can be helpful for the user training strategy and system design and can therefore improve the feasibility and operability of technical systems [13].

\subsection{LED based packaging assistant}

The second digital packaging assistant was developed (patent is pending) on the condition of using intuitive and easy-to-understand information methods (see Figure 2). Compared to the previous assistant, this variant does not need to be worn on the head or held in the hand.

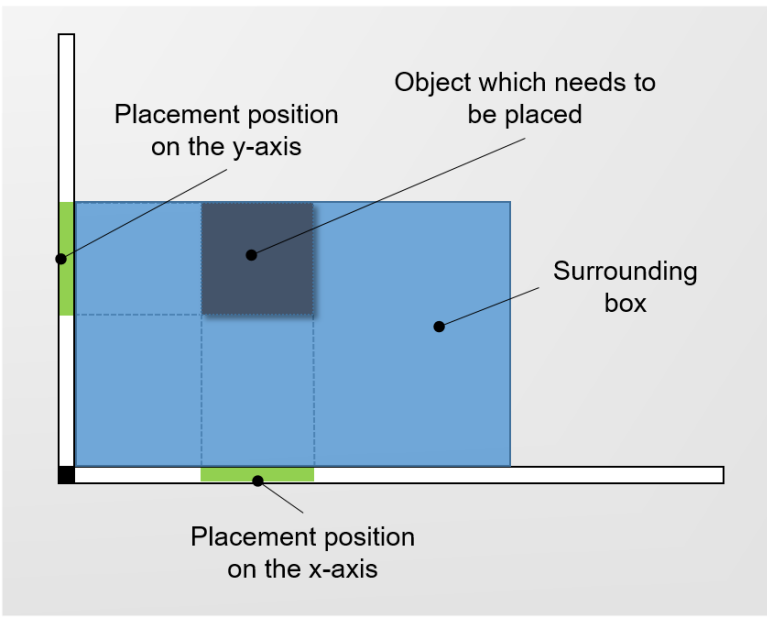

Figure 2. Functional principle of the LED assistant 
The assistant is fully integrated into the packaging desk. The information which article should be scanned and placed is given via standard display. Like in the AR assistant, gloves are used to scan the respective article. After the scanning, the information for placing the article is presented by two LED stripes attached to the packaging desk. One stripe shows the position on the $\mathrm{x}$-axis while the second stripe shows the position on the y-axis. [15]

The basic idea behind the development of this assistant was to make use of empirical knowledge that does not require an additional learning phase [16]. Based on this knowledge most people are already familiar with the assignment of colors to the respective meanings as well as the representation of data in a matrix representation with $\mathrm{x}$ - and $\mathrm{y}$-axes.

\section{Methods}

\subsection{Study sample}

All participants were recruited online. The final sample consisted of $N=28$ persons (50\% men) who were between 20 and 40 years of age $(M=26.18$, $S D=4.37)$. Participants differed in their logistics experience: $42.9 \%$ had no experience at all, the rest of the group only had theoretical $(25.0 \%)$ or both theoretical and practical logistics knowledge (32.1\%). The highest educational qualification of the sample is composed of students with a higher education entrance qualification (Abitur: German equivalent of "A Levels") (35.7\%), persons with a vocational training qualification $(7.1 \%)$ or a university degree $(57.1 \%)$. All participants had a good vision $(39.3 \%$ wearing glasses) and hearing. According to the technology competence of the sample we could conclude that subjects had a high acceptance towards technologies $(M=4.02, \quad S D=.76), \quad$ high agency $(M=4.31$, $S D=.57)$ and control beliefs $(M=3.53, S D=.64)$ and a relatively high level of the need using technology $(M=3.88, S D=.58)$. Furthermore, the subjective potential threat of technologies is rather low on average $(M=1.86, S D=.64)$.

\subsection{Procedure}

The present study evaluated the application of two technological assistance systems - AR based data glasses and a LED based packaging assistant - to indicate packaging order and scheme with a paper list as control group. Every participant was exposed to all of the three packaging devices consecutively regarding a within-subjects design. To control position and thus learning effects, the usage sequence of all three conditions AR, LED and paper list was balanced by a Latin Square design. Subjects were distributed randomly to each order.

A box $(35 \mathrm{~cm} \times 25 \mathrm{~cm} \times 14 \mathrm{~cm})$ was placed on a commercial packing table (see Figure 3). All goods to be packed were located at the table in a standardized order. Participants were asked to pack the box according to a given standardized packaging scheme via the three packaging devices. Packaging time was limited to ten minutes for each device. Participants were required to pack as many boxes as possible in this given time to create realistic working conditions under time pressure.

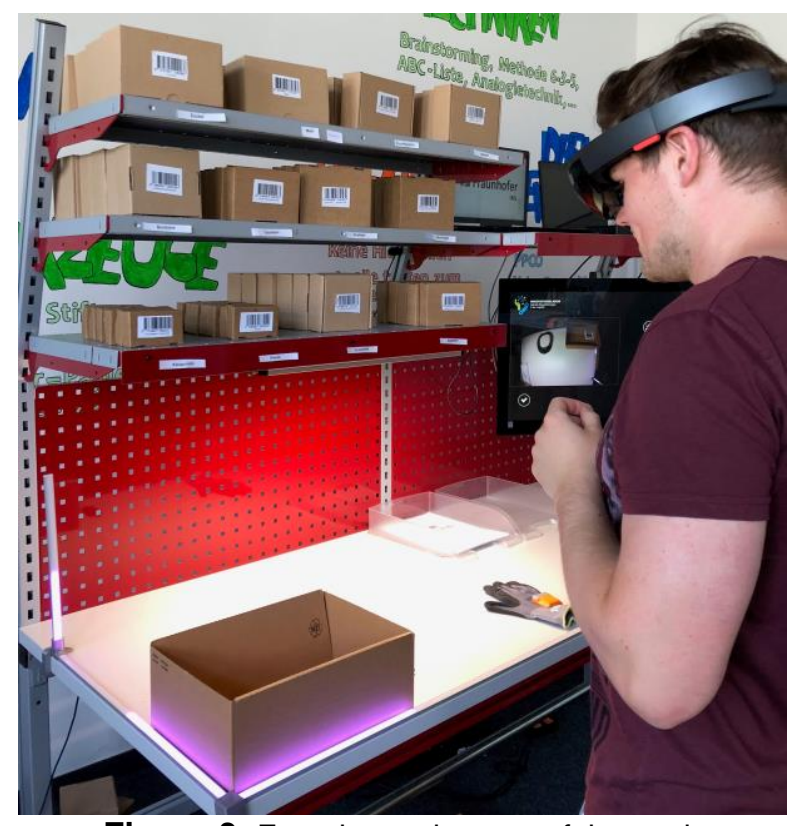

Figure 3. Experimental set-up of the study

Furthermore, participants were instructed to pack in the best possible way to simulate a real packaging process. Within the study, ten different packaging schemes were used in cyclical sequence. A total of twelve different products were available for packaging, packed in boxes of six different sizes. A packaging scheme consisted of 15 articles on average. All packaging schemes were designed in such a way that they had a comparable difficulty.

\subsection{Measurements}

After each experimental condition, several validated questionnaires were filled in to evaluate the applied device. Participants subjectively assessed the interaction with each packaging device regarding workload, usability, user experience and physical complaints. The internal consistencies of the following 
scales and subscales revealed acceptable to excellent averaged Cronbach's alpha values.

Workload. Overall workload was recorded by the NASA Task Load Index (TLX) [17] that is most widely applied to measure occurring mental costs while accomplishing system requirements [18]. The NASA TLX consists of the following six subdimensions: Mental demand, physical demand, temporal demand, performance, effort, and frustration level are rated in steps of 5 on a unipolar scale from low (0) to high (100). Total workload (raw TLX score) is the result of taking the mean value of all six subscales [18].

Usability. To measure the general appropriateness of each interface, the well-known System Usability Scale (SUS) [19] was applied. The global view of usability gives an overview of effectiveness and efficiency according to ISO 9241-11 while handling each packaging device [20]. The SUS scale consists of ten items regarding aspects such as training, complexity and need for support based on a 5-point Likert-Scale (0: strongly disagree - 4: strongly agree). By recoding several items and multiplying the sum of the scores by 2.5 the overall SUS score was calculated ranging from worst imaginable (0) to best imaginable (100) usability [21]. Additionally, one item of a German assessment tool for display workstations was applied to ask participants for the extent of appropriately displaying information via the interfaces [22]. Response format was adapted to the SUS as the original format was only dichotomous.

User experience. User experience while interacting with each packaging interface was measured with the User Experience Questionnaire (UEQ) [23]. User experience was rated with a list of 26 adjectives that had to be valued on a bipolar response format basing on a seven stage semantic differential from -3 (negative connoted adjective) to +3 (positive connoted adjective). This means that the range of the scales is between -3 (horribly bad) and +3 (extremely good). The UEQ comprises three dimensions: Attractiveness, pragmatic quality and hedonic quality. Pragmatic quality consists of the averaged values of three subscales efficiency, perspicuity and dependability and focuses on the quality of use and task-based quality. Hedonic quality is calculated by means of the two subscales stimulation and novelty and describes the design quality.

Physical complaints. A German validated questionnaire for measuring ocular and musculoskeletal strain was used [24]. Visual strain consists of seven items and musculoskeletal strain of five items. All items were rated on a 6-point rating scale from 1 (not at all) to 6 (very strong).
Sociodemographic data and technology-based personal information. Questions regarding gender, age and education were in line with the BIBB/BAuA Employment Survey 2012 [25]. To get an overview of the overall technological competence of the sample, participants filled in various scales regarding technology commitment [26] and their personal attitude towards new technologies [27]. Technology commitment consists of three subscales acceptance, control beliefs and agency beliefs according to new technologies in general (four items each). Attitudes towards technology comprise two subscales potential threat (two items) and the need for using technology (four items). The response format of every subscale was a 5-point Likert scale from 1 (strongly disagree) to 5 (strongly agree).

Objective measurements. During the experimental conditions objective measurements like the number of correctly packed boxes and error rates were measured in the given packaging time. An error was documented as soon as a packaging instruction was misinterpreted or if a carton was not packed flush with the top edge. Therefore, it can be assumed that the packaging process can be optimized by using AR glasses or a light based assistant in comparison to the traditional paper method.

\section{Results}

In the following, descriptive results regarding workload, usability, user experience and physical complaints for each packaging device are presented. Furthermore, nonparametric and therefore more powerful analyses were deployed to test mean and variance differences between the two experimental groups "AR assistant" and "LED assistant" and the control group "Paper list". Regarding statistical results probabilities of $p<.10$ are also reported besides the usual indication of $p<.05$ and $p<.01$ to show possible tendencies of the investigated differences.

\subsection{Workload}

Overall Raw TLX score. Arithmetic mean values of the Raw TLX Score and the scores of the six subdimensions mental demand, physical demand, temporal demand, performance, effort, and frustration level are shown in Figure 4.

According to the Friedman test a significant group difference of the raw TLX could be measured between all three interface conditions (Chi-Quadrat $(2)=15.71$, $p<.001)$. Results of the Wilcoxon test makes obvious that central tendencies of the Raw TLX Score differ between the AR group and the paper list group $(Z=$ 
$-3.75, p<.01, r=.72)$ as well as between the LED group and the paper list group $(Z=-3.42, p<.01, r=$ $.65)$. The overall workload was highest for dealing with the paper list while both digital assistants lead to an equal moderate workload.

Mental demand. Due to group differences in each subdimension, the mental demand score due to the LED interface $(Z=-3.82, p<.01, r=.72)$ as well as the AR interface $(Z=-3.90, p<.01, r=.75)$ differed significantly from the paper list. Mental demand when using both LED and AR did not reveal significant differences. The paper list showed the highest score while LED and AR only showed moderate results.

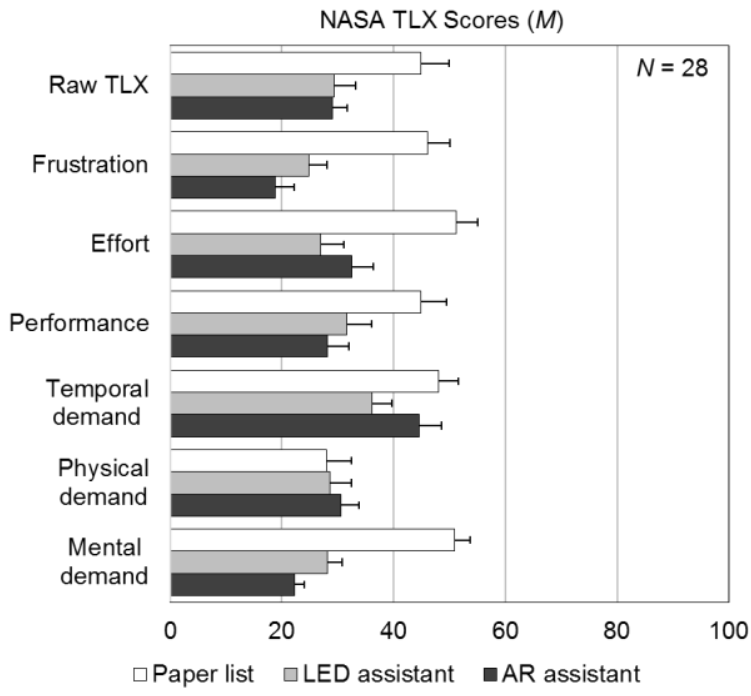

Figure 4. Descriptive statistics of the NASA TLX subdimensions and Raw TLX Score of the three packaging devices.

Physical demand. No significant group differences could be found at all for physical demands during interaction with each device. All three scenarios showed moderate results.

Temporal demand. With respect to temporal demands, there was a significant difference between the LED assistant and the paper list $(Z=-2.02, p<.05$, $r=.38)$, and between the LED assistant and the AR solution $(Z=-2.18, p<.05, r=.41)$. The LED assistant produced less temporal stress than both the AR glasses and the paper list.

Performance. Subjective perceived performance of the participants varied between the LED assistant and the paper list $(Z=-2.40, p<.05, r=.45)$ as well as between the AR glasses and the paper based device $(Z=-2.87, p<.01, r=.54)$. The best performance was evaluated for both digital packaging assistants, the worst for the paper list.

Effort. The perceived effort while using a paper list was significantly higher than using the LED assistant
$(Z=-3.16, p<.01, r=.68)$ and equally the AR solution $(Z=-3.21, p<.01, r=.61)$. The similar result can be seen according to the frustration score: This is significantly higher when using the paper list instead of the LED assistant $(Z=-2.84, p<.01, r=.54)$ or the AR glasses $(Z=-3.67, p<.01, r=.69)$. In both cases, participants valued the LED and AR solution significantly higher than the paper list.

\subsection{Usability}

Descriptive data of the overall SUS scores of the AR solution, the LED based pack assistant and the paper list are listed in Table 1.

Table 1. Descriptive statistics of the overall SUS score of the three packaging devices.

\begin{tabular}{llllll}
\hline Device & Min & Max & Median & M & SD \\
\hline Paper list & 15.00 & 95.00 & 58.75 & 60.63 & 18.40 \\
LED & 50.00 & 100 & 80.00 & 78.43 & 12.60 \\
AR & 45.00 & 90.00 & 77.50 & 72.23 & 13.61 \\
\hline
\end{tabular}

Note. $\mathrm{M}=$ Mean value, $\mathrm{SD}=$ Standard deviation.

Descriptive data indicates that the usability of the paper list and the AR solution were rated as "good" [28]. In comparison, the usability value of the LED based assistant can be interpreted as "excellent" [28].

The nonparametric Friedman test was used to examine a main effect of "device". Therefore, significant differences of variances between all three groups regarding the overall usability score could be found (Friedman-Test: $\quad$ Chi-Quadrat $(2)=13.42$, $p<.001)$.

Afterwards, the nonparametric Wilcoxon test for paired samples was deployed for detecting group differences. Results show that there is a significant difference of medians between the usability of AR and the paper list $(Z=-2.95, p<.01$, effect size according to Cohen (1992): $r=.56$ ) as well as between LED and the paper list $(Z=-3.42, p<.01, r=.66)$ and we could find a tendency of significance between the AR and LED group $(Z=-1.70, p<.10, r=.33)$. I.e. the LED assistant has the highest usability, followed by the usability of the AR solution. Usability of the paper list was valued the worst of all three devices. These results go hand in hand with the findings of the assessment of information quality that is displayed via each interface. The rating of the extent of information visualization of the LED interface tended to be better than for the AR solution $(Z=-1.69, p<.10, r=.32)$. The evaluation of the information quality displayed via the AR glasses reached higher ratings than for the paper list $(Z=-3.57$, $p<.001, r=.67)$. 


\subsection{User Experience}

In Figure 5 descriptive results (means, standard errors) of the six user experience subscales attractiveness, stimulation, novelty, efficiency, perspicuity, and dependability are given for each packaging interface.

User experience values between -.8 and .8 represent a neutral evaluation of the corresponding scale (white color), values $>.8$ represent a positive evaluation (light grey shading) and values $<-.8$ represent a negative evaluation (dark grey shading) [23].
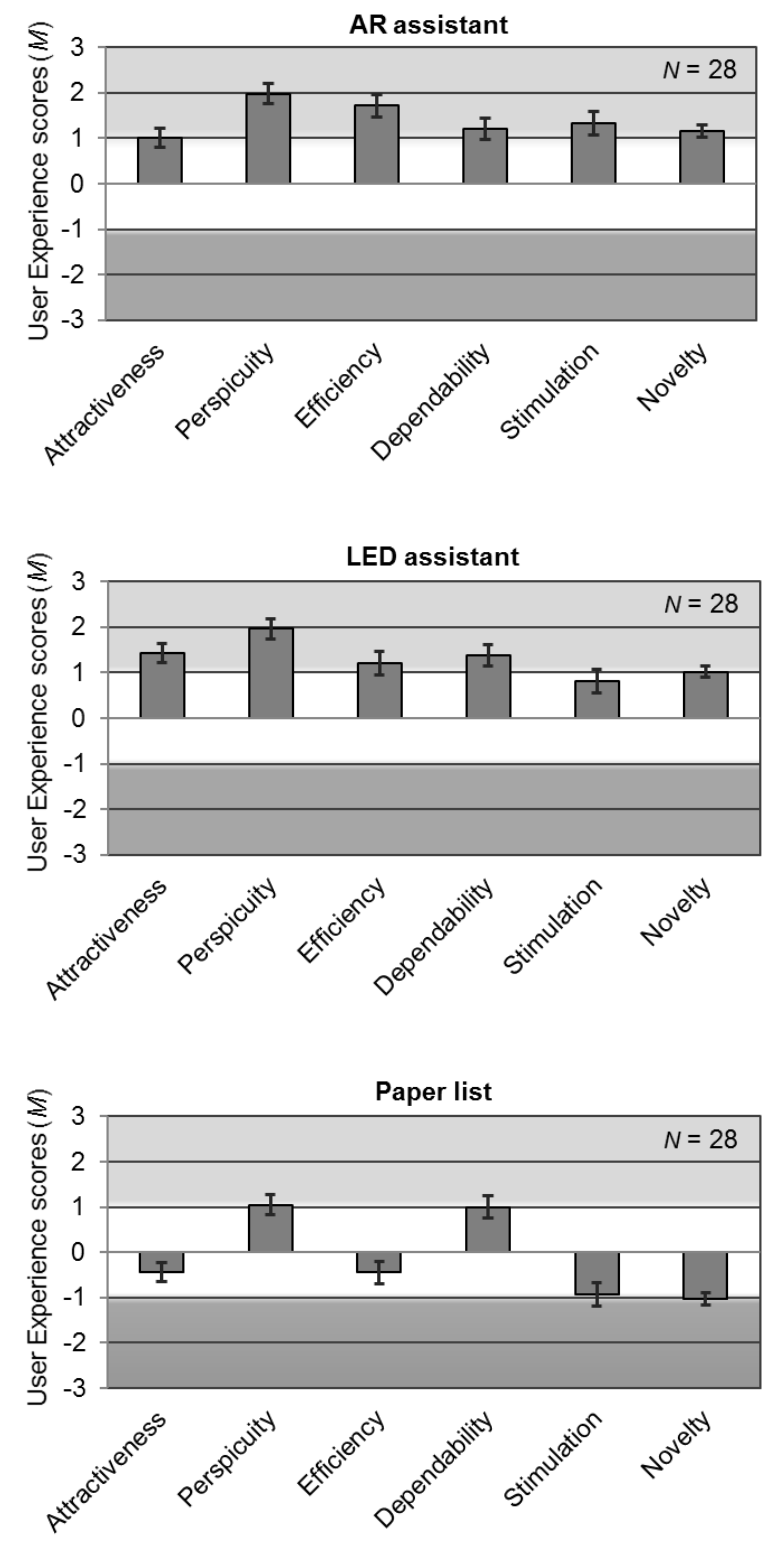

Figure 5. User experience scores for the AR assistant, LED assistant and the paper based packaging device.
First bar diagram in Figure 5 shows that regarding the AR pack solution all sub-dimensions were positively assessed. Equally, according to the LED packaging assistant all the subscales were rated positively (see Figure 5, second bar diagram). With respect to the paper list, only perspicuity and dependability were evaluated as positive (see Figure 5, third bar diagram). Attractiveness and efficiency reached a moderate evaluation, whereas the subscales stimulation and novelty were assessed as negative.

Subscales can be classified into the three dimensions attractiveness, pragmatic quality (perspicuity, efficiency, dependability) and hedonic quality (stimulation, novelty). Attractiveness, pragmatic quality and hedonic quality of each packaging interface are presented in Table 2.

Table 2. Descriptive statistics of the grouped user experience subscales attractiveness, pragmatic quality (PQ) and hedonic quality (HQ) of all packaging devices.

\begin{tabular}{lllllll}
\hline Device & \multicolumn{2}{c}{ Attractiveness } & \multicolumn{2}{c}{ PQ } & \multicolumn{2}{c}{ HQ } \\
& $\mathrm{M}$ & SD & M & SD & M & SD \\
\hline Paper list & -.44 & 1.09 & .54 & .99 & -.98 & 1.01 \\
LED & 1.42 & .95 & 1.51 & .76 & .92 & .85 \\
AR & 1.01 & .94 & 1.64 & .66 & 1.25 & .58 \\
\hline Note $M=$ &
\end{tabular}

Note. $M=$ Mean value, $S D=$ Standard deviation.

With respect to the Friedman test a significant main effect "device" regarding attractiveness could be detected (Chi-Quadrat $(2)=20.94, p<.001)$. Results of the Wilcoxon test indicate that central tendencies of attractiveness differ between the AR group and the paper list group $(Z=-3.77, p<.001, r=.71)$ as well as between the LED group and the paper list group $(Z=$ -4.27, $p<.001, r=.81)$. Between both digital assistants we could find a tendency of a significant difference $(Z=-1.70, p<.10, r=.32)$.

According to the pragmatic quality a significant group difference was also found (Chi-Quadrat $(2)=$ 14.25, $\quad p<.01)$. More precisely, both digital devices differentiated significantly from the paper based interface (AR versus paper list: $Z=-3.69, p<.001$, $r=.71$; LED versus paper list: $Z=-3.76, p<.001$, $r=.70$ ). Furthermore, the AR interface did not vary significantly from the LED interface. Equally, due to the hedonic quality a significant main effect of "device" was measured (Chi-Quadrat $(2)=36.56$, $p<.001)$. The Wilcoxon test clarifies a significant difference between each packaging method (AR versus paper list: $Z=-4.49, p<.001, r=.85$; LED versus paper list: $Z=-4.19, p<.001, r=.79$; AR versus LED: $Z=-2.13, p<.05, r=.40)$.

Results indicate that both digital assistants are attractive packaging methods that also have a high pragmatic and hedonic quality quality. In detail, the 
LED assistant tends to be more attractive for participants than the AR solution. Additionally, the LED interface reached the best assessment regarding hedonic quality. In comparison, the paper based packaging list was only characterized by a moderate attractiveness and pragmatic quality. Furthermore, the result for design quality of the paper list was only at a negative level. Therefore, the paper based interface had the worst values regarding attractiveness, pragmatic quality and hedonic quality.

\subsection{Physical complaints}

Visual strain. Arithmetic mean value of the ocular strain for the AR assistant was in total at a low level $(M=1.99, S D=.72)$. It is noteworthy that ratings of various items varied between "no complaints" (Min = 1) and "severe complaints" (Max =6).

Musculoskeletal strain. Regarding both digital assistants musculoskeletal strains were queried. Results indicate that mean values were at a low level (AR: $M=$ 2.14, $S D=.93 ;$ LED: $M=1.63, S D=.71)$. Musculoskeletal strain was significantly higher while using the AR assistant than the LED interface $(Z=$ $-3.23, p<.01, r=.62)$.

\subsection{Objective measurements}

Number of packed boxes. Overall performance was comparably high for all three packaging interfaces. Most often, three or four boxes were packed (AR: $M=$ 3.82, $S D=.91$; LED: $M=3.46, S D=.69$; Paper list: $M=3.75, S D=1.43$ ).

Error rates. Since a zero error rate is decisive in logistics, we analyzed the individual absolute values of the test persons when evaluating the error rates. It becomes clear that when looking at the absolute error values, differences in the experimental conditions can be observed. Of 28 test persons, both AR and LED 23 persons carried out the packaging process completely without any errors (82\%). Four test persons had each made one error and one test person two errors. When using the paper-based packing list, 16 out of 28 test persons carried out the process without any errors (57\%). Six participants made one error, five made two errors and one participant made seven errors.

\section{Discussion and Outlook}

\subsection{Summary and Conclusion}

In this paper we introduced two different digital assistance systems to indicate packaging order and scheme: AR based data glasses and a LED based packaging assistant. The collaboration between human and these devices was investigated in a laboratory study. Human-centered factors such as subjective workload, usability, user experience, physical complaints and objective measurements were evaluated in comparison to a paper based interface as control group. Results indicate that both digital packaging interfaces are appropriate solutions to assist warehouse workers in packaging.

It can be concluded that the AR and LED interface may cause a lower overall workload than a conventional paper based list. Both digital devices did not differ in various workload sub-dimensions that were only at a low to moderate level. It was particularly noticeable that the LED interface produced less temporal stress than the AR device. Furthermore, results imply that usability was assessed best for the LED interface with an excellent rating, while usability for the AR interface got a good rating. Concluding, the AR device is already a suitable solution for packaging but should be further optimized. That goes along with the user experience results. Both digital interfaces seem to be appropriate for packaging as the pragmatic quality is comparably good. The good pragmatic quality of the AR and LED solution also becomes recognizable in low error rates and high self-reported performance. Although attractiveness and hedonic quality of both digital interfaces had a positive evaluation, the LED device tended to be more attractive and reached the best assessment according to hedonic quality. According to physical complaints, results make clear that the LED interface seems less demanding than the AR device, especially visual and musculoskeletal strain. All in all, along with digitization it becomes obvious that technology assistance systems have a great potential to assist in intralogistics namely in the packaging area.

\subsection{Industrial Application}

Based on the results of the study, it can be said that both assistance systems are suitable for use in the chosen scenario. However, a distinction must be made at this point between the AR solution and the LED solution. The AR glasses represent information in an intuitive way, but do not yet meet the ergonomics and performance requirements required in industry. Based on the feedback of the participants regarding the AR solution, it becomes clear that the presentation of information embedded in reality is the most intuitive way of providing information. This can be explained by the fact that the virtual information could be directly linked visually with the associated physical object. This would avoid misunderstandings and improves the 
perspicuity. That goes along with the results of user experience (see Figure 5).

On the other hand, the LED solution shows that information can also be displayed intuitively without great effort. Just by using light and different colours, similar results can be achieved like with AR glasses. The basic requirement for the industrial use of digital assistants for packaging is that a software system is used that carries out the optimization and makes it available to the assistant. However, at this point it must be limited that the LED solution is only suitable for applications with shallow depth. This system might lose accuracy at greater depths. An example of this is the loading of trucks or containers with packages.

Compared to the paper-based solution, the study did not show any major differences. Furthermore, the number of error-free runs with the digital assistants was almost $44 \%$ higher than with the paper-based process. Also, the variance in the number of fully packed boxes was greater when using paper. It became clear that the frequency of errors and the variance of quality in production use can be considerably reduced by digital assistants. The higher mental requirement for packaging with a paper list increases the risk that the performance of the worker will decrease considerably. This is not to be expected with the digital assistants, because the mental load was considerably lower.

\subsection{Limitations and Future Research}

As technology competence of the sample was at a high level, it can be supposed that the results may represent only individuals which are attracted by the possibility to try out new technologies. In further studies, the study's topic should be kept secret. Another limitation of our study is that all participants had a high education level. Thus, the transfer of our results to the warehouse industry could be difficult. In further studies, digital assistants for packaging should be tested by real warehouse workers. This could increase the external validity of our study results. Furthermore, against previous study results, ten minutes of packaging are already a good study condition, but we only can suppose long-term effects on workload or physical strain while handling AR or LED assistants. Further, it would be interesting to measure individual stress physiologically according to previous studies [29]. Thus, we could enrich our study with objective measurements and we could conclude if there is also an effect on vital parameters that indicate stress such as heart rate variability.

In future analyses, it is planned to analyse workload, usability and user experience for gender, age and also technology competence effects. It can be supposed that workload may increase when technology acceptance is low or perceived control or agency beliefs are low. Furthermore, we will proof if there is a significant correlation between usability, user experience and workload - also independent of covariates like age or gender. All in all, we intend to provide recommendations for deploying and designing collaboration technologies in compliance with cognitive aspects, especially in the intralogistics sector.

\section{Acknowledgements}

This study was founded by the »Innovationlab Hybrid Services in Logistics" and the »Centre of Excellence for Logistics and IT «. We thank Felix Bedarf, Alexandra Eichler, Hermann Foot, Friederike Hering and David Kloth for supporting us in organizing and conducting the evaluation study. A special thank goes to Désirée Bullock for her support in proofreading our paper.

\section{References}

[1] Reif, R., and Walch, D., "Augmented \& Virtual Reality applications in the field of logistics", in: The Visual Computer, 24 (11), Springer, Berlin Heidelberg, 2008, pp. 987-994.

[2] Bundesverband Paket und Expresslogistik, Wachstum über Grenzen hinweg, BIEK, Berlin, 2017.

[3] Tüllmann, C., ten Hompel, M., Nettsträter, A., and Prasse, C., "Social Networked Industry ganzheitlich gestalten", in: Future Challenges in Logistics and Supply Chain Management, vol. 6, Fraunhofer IML, Dortmund, 2017.

[4] Kretschmer, V., Eichler, A., Rinkenauer, G., and Spee, D., "Focus on the human being: Cognitive Ergonomics in intralogistics", in: Logistics Journal: Proceedings, vol. 2017, 2017, pp. 1-6.

[5] Capgemini, The current and future state of digital supply chain transformation, 2016, p. 5.

[6] Grosse, E. H., Glock, C. H., Neumann, W. P., "Human factors in order picking: a content analysis of the literature", in: International Journal of Production Research, 55 (5), 2017, pp. $1260-1276$.

[7] Trivella, A., Pisinger, D., Bin-packing problems with load balancing and stability constraints, in: INFORMS Transportation and Logistics Society, 2017.

[8] Ibáñez, M.-B., Delgado-Kloos, C., “Augmented reality for STEM learning: A systematic review", in: Computers \& Education, vol: 123, 2018, pp. 109-123. 
[9] Maettig B., Lorimer I., Jost J., Kirks T., "Analysis of the application of Augmented Reality in the packaging process considering specific requirements on the visualization of information as well as the ergonomic integration of humans into the process", in: Logistics Journal: Proceedings, vol. 2016, 2016, pp. 97-106.

[10] Jost, J., Kirks, T., Maettig, B., "Study on manual palletization of inhomogeneous boxes with the help of different interfaces to assess specific factors of ergonomic impact", in: Proceedings of the 19th International Conference on Human-Computer Interaction with Mobile Devices and Services (MobileHCI '17), ACM, New York, Article no. 53, 2017.

[11] Kretschmer, V., Plewan, T., Rinkenauer, G., \& Maettig, B., "Smart palletisation: Cognitive Ergonomics in Augmented Reality based palletising", in: International Conference on Intelligent Human Systems Integration, Springer, Cham, 2018, pp. 355-360.

[12] Quandt, M., Knoke, B., Gorldt, C., Freitag, M., Thoben, K.-D., „General Requirements for Industrial Augmented Reality Applications", in: Procedia CIRP, 72, 2018, pp. 1130-1135.

[13] Wu, H., Liang, S., Hang, W., Liu, X., Wang, Q., Choi, K.-S., Qin, J., „Evaluation of motor training performance in 3D virtual environment via combining brain-computer interface and haptic feedback", in: Procedia Computer Science, vol. 107, 2017, pp. 256-261.

[14] Wertheimer, M., Laws of organization in perceptual forms, in: W. D. Ellis (Ed.), A source book of Gestalt psychology, London, 1938, pp. 71-88.

[15] Maettig, B., Hering, F., Doeltgen, M., "Development of an intuitive, visual packaging assistant", in: I. L. Nunes (ed.), Advances in Human Factors and Systems Interaction. Proceedings of the AHFE 2018 International Conference on Human Factors and Systems Interaction, July 21-25, 2018, Loews Sapphire Falls Resort at Universal Studios, Orlando, Florida, USA, Springer, 2018, pp 19-25.

[16] Anderson, R. (Ed.), Spiro, R. (Ed.), Montague, W. (Ed.), Schooling and the Acquisition of Knowledge, London, Routledge, 1977.

[17] Hart, S. G., and Staveland, L. E., "Development of NASA-TLX (Task Load Index): Results of empirical and theoretical research", in: Hancock, P.A. and Meshkati, N. (eds.), Human mental workload, Elsevier, Amsterdam, 1988, pp. 139-183.

[18] Hart, S.G., "Nasa-Task Load Index (Nasa-TLX); 20 Years Later", in: Proceedings of the Human Factors and Ergonomics Society Annual Meeting, vol. 50, 2006.

[19] Brooke, J., "SUS: A "quick and dirty" usability scale", in: Jordan, P.W., Thomas, B., Weerdmeester, B.A. and
McClelland, A.L. (eds.), Usability Evaluation in Industry, Taylor and Francis, London, 1996.

[20] International Organization for Standardization, ISO 9241-11: Ergonomic Requirements for Office Work with Visual Display Terminals (VDTs): Part 11: Guidance on Usability, ISO, Freiburg i. B., 1998.

[21] Brooke, J., "SUS: A Retrospective", in: Journal of Usability Studies, vol. 8, 2013, pp. 29-40.

[22] ASER, „BiFra - Bildschirm-Fragebogen. Ein Beurteilungsinstrument für Bildschirmarbeitsplätze zur Umsetzung des Arbeitsschutzgesetzes und der Bildschirmarbeitsverordnung“, Institut ASER, Wuppertal, 1998.

[23] Laugwitz, B., Held, T., and Schrepp, M., "Construction and Evaluation of a User Experience Questionnaire", in: Holzinger, A. (eds.), HCI and Usability for Education and Work: Proceedings of the 4th Symposium of the Workgroup Human-Computer Interaction and Usability Engineering of the Austrian Computer Society, USAB 2008, Graz, Austria, November 20-21, Springer, Berlin Heidelberg, 2008, pp. 6376.

[24] Jaschinski, W., König, M., Mekontso, T. M., Ohlendorf, A., and Welscher, M., „Computer vision syndrome in presbyopia and beginning presbyopia: Effects of spectacle lens type", in: Clinical and Experimental Optometry, 98 (3), Lippincott Williams \& Wilkins, 2015, pp. 228-233.

[25] Wittig, P., Nöllenheidt, Ch., and Brenscheidt, S., Grundauswertung der BIBB/BAuA-Erwerbstätigenbefragung 2012. mit den Schwerpunkten Arbeitsbedingungen, Arbeitsbelastungen und gesundheitliche Beschwerden, BAuA Bundesanstalt für Arbeitsschutz und Arbeitsmedizin, Dortmund, 2013.

[26] Neyer, F. J. J., Felber, J., and Gebhardt, C., Kurzskala. Technikbereitschaft (TB) [Technology commitment], in: ZIS - Zusammenstellung sozialwissenschaftlicher Items und Skalen (ed.), 2016.

[27] Claßen, K., Zur Psychologie von Technikakzeptanz im höheren Lebensalter: Die Rolle von Technikgenerationen. Dissertation, Heidelberg, 2013.

[28] Bangor, A., Miller, J., and Kortum, P., "Determining What Individual SUS Scores Mean: Adding an Adjective Rating Scale", Journal of Usability Studies, 4 (3), 2009, pp. 114-123.

[29] Mättig, B., Döltgen, M., Archut, D., Kretschmer, V., "Intelligent work stress monitoring: Prevention of workrelated stress with the help of physiological data measured by a sensor wristband. Proceedings of the Intelligent Systems Conference (IntelliSys 2018), September 6-7, London, UK, IEEE, 2018, pp. 1304-1309. 